\title{
ANÁLISE DO FLUXO EM MOLDE DE LINGOTAMENTO CONTÍNUO DE BEAM BLANK COM O USO DE APENAS UMA VALVULA TUBULAR*
}

\section{Resumo}

Weslei Viana Gabriel Johne Jesus Mol Peixoto ${ }^{1}$ Leticia Queiroz Ribeiro ${ }^{2}$ Samuel da Silva de Souza ${ }^{2}$ Carlos Antonio da Silva ${ }^{3}$ Itavahn Alves da Silva ${ }^{3}$ Varadarajan Seshadri

O fluxo simétrico e distribuição de temperatura uniforme são de grande importância no processo de Lingotamento Contínuo de Beam Blank, porém a utilização de duas válvulas para alimentação do molde traz dificuldades operacionais relacionadas ao posicionamento das válvulas e controle de vazão. Neste sentido é interessante trabalhar com apenas uma válvula. Neste trabalho técnicas de modelagem física e matemática foram utilizadas para avaliar o fluxo de fluido no molde, comparando-se o caso em que se empregam duas válvulas tubulares, com aquele em que se utiliza apenas uma válvula. Verificou-se que esta última configuração resultou em uma mudança no comportamento do fluxo, aumento na profundidade de impacto, porém a velocidade na região do menisco não teve variação significativa em comparação com a utilização de duas válvulas tubulares.

Palavras-chave: Beam blank; Fluxo; Lingotamento contínuo; Válvula tubular.

\section{FLUID FLOW ANALYSIS IN MOULD FOR BEAM BLANK CONTINUOUS CASTING USING ONLY ONE STRAIGHT TUBULAR SEN}

\section{Abstract}

An uniform temperature distribution and fluid flow symmetry are of great importance for Beam Blank Continuous Casting process. However, the application of two SEN (Submerged Entry Nozzle) can result in operational problems related to the SEN positioning as well as flow rate control. For this reason, it becomes interesting to work with just one SEN. In this work physical and mathematical modeling are used to describe the fluid flow inside the mold. The cases of one Straight Tubular SEN and two Straight Tubular SEN are compared. It was verified that the former leads to an increase in impact depth, however the velocity at meniscus did not change significantly.

Keywords: Beam blank; Continuous casting; SEN; Fluid flow.

1 Engenheiro Metalúrgico, Mestrando em Engenharia de Materiais, Redemat, Universidade Federal de Ouro Preto, Ouro Preto, Minas Gerais, Brasil.

2 Graduando em Metalurgia, Escola de Minas, Ouro Preto, Minas Gerais, Brasil.

3 Engenheiro Metalúrgico, Ph.D., Professor, Universidade Federal de Ouro Preto, Escola de Minas, Ouro Preto, Minas Gerais, Brasil.

4 Engenheiro Metalúrgico, Dr. Ing., Professor Emérito, Universidade Federal de Minas Gerais (UFMG), Belo Horizonte, Minas Gerais, Brasil. 


\section{INTRODUÇÃO}

A principal configuração de válvulas submersas para o lingotamento contínuo de beam blank é formada por duas válvulas tubulares posicionadas no centro do flange. Visando melhorar a qualidade do produto lingotado, outros modelos de válvulas têm sido estudados. Chen et al. [1] e Yang et al. [2] analisaram o fluxo para uma nova configuração de válvulas submersas composta por três furos laterais com ângulo de $120^{\circ}$ entre eles e encontraram condições que melhoram a limpidez do aço devido a redução na profundidade de impacto do jato aliada a melhoria nas condições de fusão do pó fluxante.

Porém, nestes estudos foi considerada a utilização de duas válvulas submersas, sendo que esta condição proporciona dificuldades operacionais como a necessidade de duplo controle de vazão e de garantir simetria no posicionamento das válvulas nos flanges. Uma pequena variação de posição ou de vazão entre as válvulas altera o padrão de fluxo, alterando a distribuição de temperaturas na seção transversal, podendo acarretar em entranhamento de escória, pontos quentes, redução da uniformidade da pele solidificada, formação de trincas, ou até mesmo em breakouts.

Para contornar estas dificuldades operacionais, uma alternativa é estudar a possibilidade de trabalhar com apenas uma válvula submersa. De Santis et al. [3] foram pioneiros em propor uma configuração composta de apenas uma válvula para alimentação do molde, sendo o modelo formado por dois furos circulares na saída da válvula, o furo maior direcionado para o flange oposto e o menor para baixo (Figura 1).

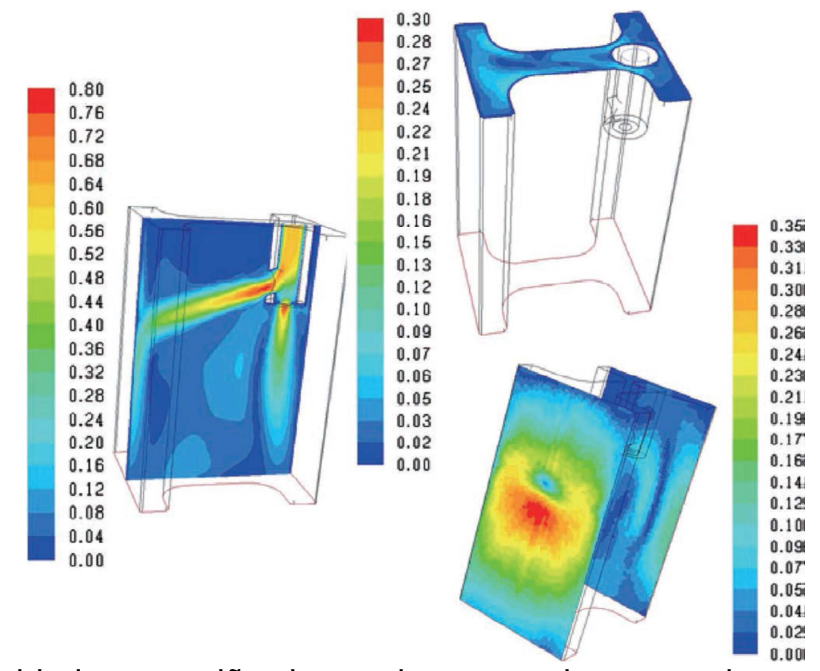

Figura 1. Perfil de velocidade na região do menisco, paredes e no plano central para o modelo de válvula proposto por De Santis et al. [3].

Ao definir novos modelos de alimentação, é necessário descrever o fluxo do fluido, uma vez que isto irá interferir no fluxo de calor e consequentemente na formação da casca sólida. Lee et al. [4] verificaram que na região em que se tem contato do jato com a casca formada, a velocidade de crescimento da mesma é reduzida. Além disto se observa variação na troca térmica ao longo da seção, o que promove mudança na espessura da casca solidificada, formando gaps de ar em determinadas regiões, como nas quinas dos flanges, e estes gaps aumentam a resistência ao fluxo de calor e consequentemente elevam a temperatura, gerando concentração de tensão que podem resultar na formação de trincas ou outros defeitos, como até mesmo breakouts, conforme verificaram Lee et al. [4] e Hibeller et al. [5]. 
Trabalhos descrevendo, por modelagem física e matemática, a estrutura de fluxo no interior de um molde de Beam Blank são escassos. As consequências de utilização de apenas uma válvula tubular não são plenamente discutidas. Este trabalho tem o objetivo de descrever o padrão de fluxo para esta ultima configuração e compará-lo com o fluxo existente quando se utiliza duas válvulas tubulares.

\section{MATERIAIS E MÉTODOS}

Foram realizadas simulações físicas e matemáticas do fluxo de fluido no interior de um molde com formato de beam blank, avaliando a velocidade e profundidade do jato, assim como campo vetorial.

Nas simulações físicas utilizou-se modelo em acrílico escala 1:1 (Figura 2.a), de um molde de beam blank com dimensões $499 \mathrm{mmX} 415 \mathrm{mmX} 125 \mathrm{~mm}$ e altura de $1,5 \mathrm{~m}$, e água como fluido de trabalho a frio para simular o fluxo de aço. Os testes foram realizados com válvula tubular cujo diâmetro interno é de 53,2mm (válvula Tipo A). As vazões utilizadas foram de $100 \mathrm{~L} / \mathrm{min}, 125 \mathrm{~L} / \mathrm{min}$ e $150 \mathrm{~L} / \mathrm{min}$, que correspondem às velocidades de lingotamento de $0,78 \mathrm{~m} / \mathrm{min} ; 0,98 \mathrm{~m} / \mathrm{min}$ e $1,2 \mathrm{~m} / \mathrm{min}$ respectivamente, com profundidade de imersão igual a $100 \mathrm{~mm}$. Comparou-se a variação de velocidade em uma das regiões do menisco e a profundidade do jato com as de um teste realizado usando duas válvulas tubulares com $26,7 \mathrm{~mm}$ de diâmetro (válvula Tipo B).

Os fluxos foram analisados em três áreas do molde perpendiculares ao menisco, possibilitando descrevê-lo na região da alma (plano de simetria), região do flange que contém a válvula (sendo que o plano analisado passa pelo centro da válvula) e do flange situado no lado oposto a esta (plano com $25 \mathrm{~mm}$ de distância da parede do flange). A posição das regiões analisadas pode ser verificada através da Figura 2.(b) na qual o plano que passa pelo centro da válvula é o Plano 1, enquanto o plano localizado a 2,5cm da face larga do flange oposto à válvula é o Plano 2.

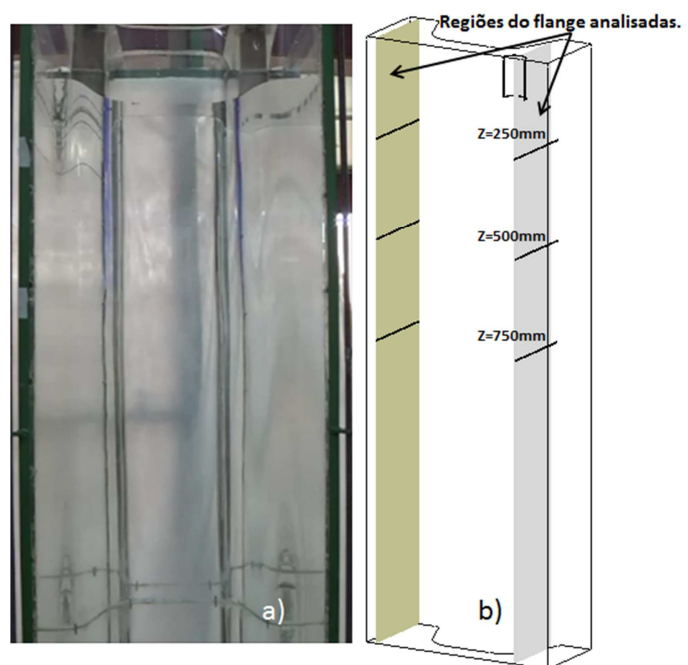

Figura 2. a) Molde em acrílico utilizado nas simulações físicas; b) representação dos planos, Plano 1 e Plano 2, utilizados nas análises dos resultados da simulação computacional.

Para avaliação do campo vetorial usou-se os ensaios em que incidiu-se um plano de laser sobre uma determinada área de interrogação iluminando partículas (com densidade igual a da água) contidas no fluido. Estas partículas acompanham o fluxo do fluido permitindo, portanto, a identificação da trajetória. 
Para análise quantitativa do fluxo por meio de simulação física foi utilizada a técnica PIV (Particle Image Velocimetry) através do aparelho fornecido pela DANTEC ${ }^{\circledR}$ existente no laboratório de Pirometalurgia do DEMET-UFOP. Esta técnica consiste basicamente em seguir o fluxo das partículas dispersas no fluido em um plano gerado pela iluminação com o laser Sheet Visualization e captação de imagens na filmagem deste plano por uma câmera CCD (Figura 4).

As imagens, geradas a partir de dois pulsos sucessivos do laser, são processadas em software específico e a velocidade (além de linhas de fluxo, vorticidade, valores médios e flutuações) é determinada a partir de um processo de interpolação. Foi avaliado o campo vetorial da altura do menisco até uma profundidade de aproximadamente $90 \mathrm{~cm}$ permitindo a análise do fluxo e do redemoinho.

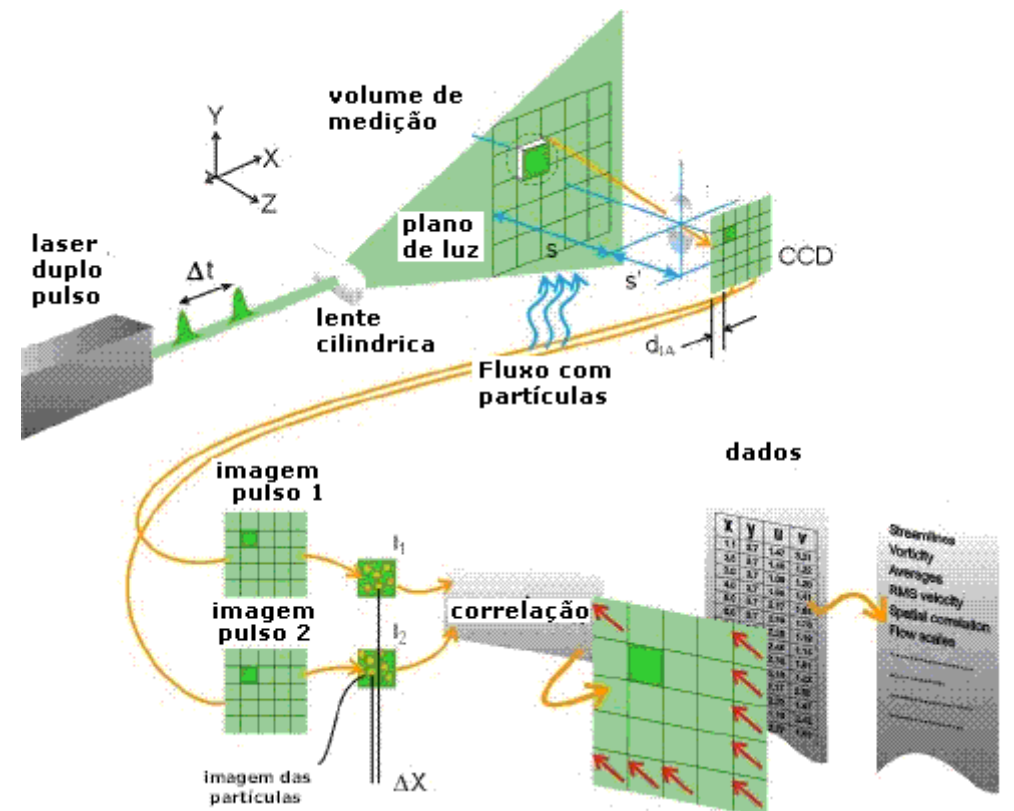

Figura 3. Esquema de funcionamento de um aparelho PIV, de acordo com a DANTEC®.

As simulações computacionais foram realizadas em regime permanente utilizandose o software Ansys ${ }^{\circledR}$ CFX 15.0, considerando a água um fluido Newtoniano, temperatura constante e igual a $25^{\circ} \mathrm{C}$. O modelo aplicado foi o $\mathrm{k}-\epsilon$, no qual se resolve as equações da continuidade, de Navier Stokes e da Viscosidade efetiva, além das equações auxiliares para determinação de $k$ (energia cinética de turbulência) e $€$ (taxa de dissipação de energia cinética). Em função das condições de simetria e visando reduzir o tempo de análise, a simulação foi realizada considerando metade do molde conforme se verifica na Figura 2-(b).

Condições de contorno aplicáveis ao problema:

- Paredes - Condição de não deslizamento nas paredes do molde;

- Condição de livre deslizamento no menisco;

- Vazão mássica na entrada - 1,667kg/s; 2,083kg/s e 2,5kg/s para as velocidades de lingotamento equivalentes a $0,78 \mathrm{~m} / \mathrm{min} ; 0,98 \mathrm{~m} / \mathrm{min}$ e $1,2 \mathrm{~m} / \mathrm{min}$ respectivamente.

- Condição de simetria no plano que corta o centro da alma;

Os resultados obtidos através da simulação matemática são validados quando apresentam resultados semelhantes aos de simulação física, isto é determinado através da comparação dos mapas vetoriais obtidos em ambas as técnicas, assim como pelos valores de velocidades. 


\section{RESULTADOS E DISCUSSÃO}

Os resultados foram divididos em três tópicos em função das variáveis analisadas.

\subsection{Análise do Campo de Velocidades}

A figura 4 apresenta uma comparação entre os mapas vetoriais obtidos via simulação computacional (Ansys CFX) e através da técnica PIV para o plano 1. Note que nesta última o mapa vetorial encontra-se subdividido em três partes. Devido a restrições na área de captura de imagens pelo PIV, com relação às dimensões do modelo, foi necessário subdividir o modelo em três regiões analisando-as separadamente, sendo a altura analisada em cada teste de $30 \mathrm{~cm}$. Como se nota existe semelhança quanto à estrutura do jato nas duas simulações.

Avaliou-se os valores de velocidade ao longo da metade da face estreita do molde no plano 1 , nas distâncias ao menisco de $250 \mathrm{~mm}, 500 \mathrm{~mm}$ e $750 \mathrm{~mm}$, conforme Figura 2.b. Os resultados de componente vertical de velocidade, tanto de simulação física quanto computacional estão apresentados na Figura 5. Nota-se boa concordância entre as simulações, o que valida a simulação computacional para esta configuração de válvula submersa.

A velocidade é maior na região central do flange, junto à válvula, em função da força de impacto do jato oriundo da válvula. Com o aumento da velocidade de lingotamento, aumenta-se tanto a velocidade do jato (descendente) quanto à velocidade do fluido que retorna no flange oposto (ascendente).

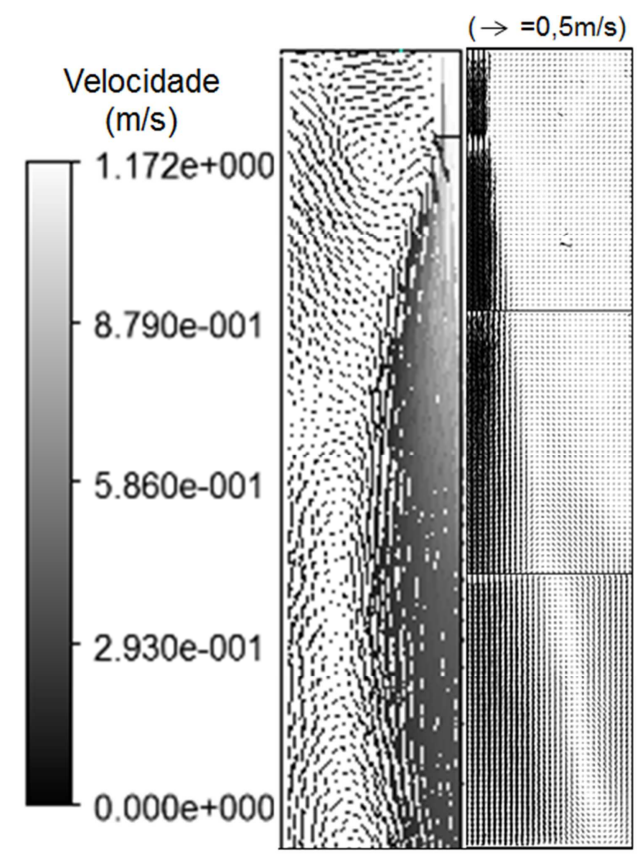

(a) CFX

(b) PIV

Figura 4. Mapa vetorial no plano 1 para uma vazão de $125 \mathrm{~L} / \mathrm{min}$ obtido via: (a) simulação computacional e (b) simulação física - técnica PIV. 

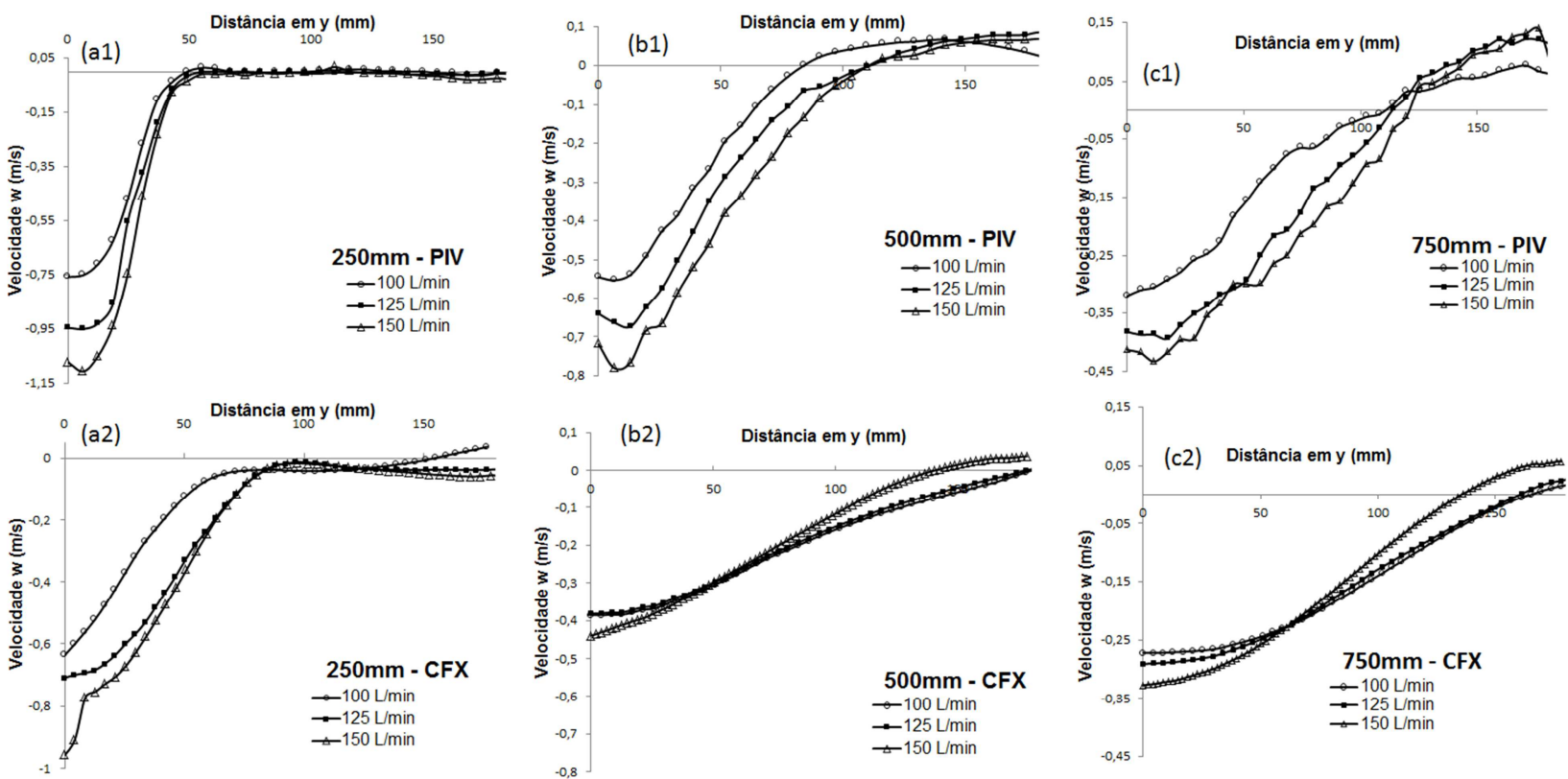

Figura 5. Gráficos de velocidade em função da distância y do centro da válvula até o filete da face estreita do flange que contém a válvula: Técnica PIV a uma distância do menisco de a1) 250mm, b1) $450 \mathrm{~mm}$ e c1) $750 \mathrm{~mm}$; Simulação numérica no CFX a uma distância do menisco de a2) $250 \mathrm{~mm}$, b2) $500 \mathrm{~mm}$ e c2) $750 \mathrm{~mm}$.

A figura 6 apresenta os valores de velocidade no plano 2 obtidos pela técnica PIV a $250 \mathrm{~mm}, 500 \mathrm{~mm}$ e $750 \mathrm{~mm}$ do menisco respectivamente. Percebe-se, como esperado, valores de velocidade menores que os obtidos no plano 1 . Com o aumento da distância com relação ao menisco aumenta-se a velocidade devido a aproximação ao ponto de recirculação do fluido. A $250 \mathrm{~m}$ do menisco o decaimento no valor de velocidade próximo ao centro do flange pode ser explicado em função do vórtice apresentado nesta região. Estes resultados estão de acordo com a simulação computacional.
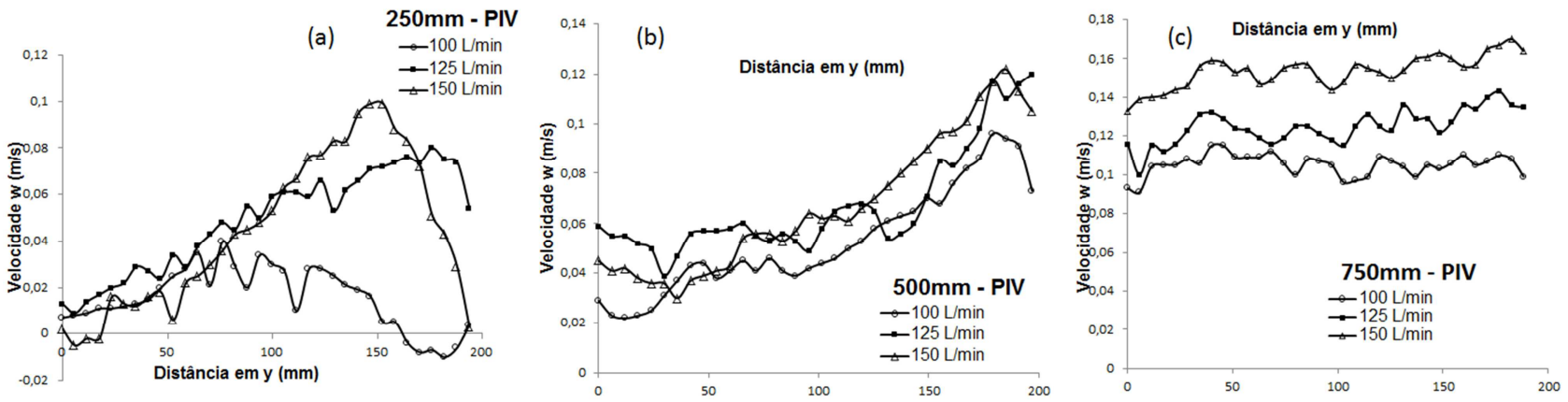

Figura 6. Gráficos de velocidade em função da distância y em três linhas no plano 2 obtidos pela técnica PIV a uma distância do menisco de a) $250 \mathrm{~mm}$, b) $500 \mathrm{~mm}$ e c) $750 \mathrm{~mm}$.

\subsection{Profundidade do Jato}

Para as vazões de $100 \mathrm{~L} / \mathrm{min}, 125 \mathrm{~L} / \mathrm{min}$ e $150 \mathrm{~L} / \mathrm{min}$ os valores de profundidade do jato foram $0,92 \mathrm{~m}, 1,19 \mathrm{~m}$ e $1,29 \mathrm{~m}$ respectivamente, quando se empregou apenas uma válvula tubular de $53,2 \mathrm{~mm}$ de diâmetro. Estes valores são maiores que a altura real do molde $(0,8 \mathrm{~m})$. Quando se utilizou duas válvulas tubulares com diâmetro interno de $26,7 \mathrm{~mm}$ as profundidades do jato foram $0,76 \mathrm{~m}, 0,81 \mathrm{~m}$ e $0,84 \mathrm{~m}$ respectivamente para mesmas vazões citadas. Chen et al. [6], em análise usando 
duas válvulas tubulares encontraram para uma velocidade de lingotamento de $0,9 \mathrm{~m} / \mathrm{min}$, uma profundidade de impacto de $1,09 \mathrm{~m}$. Eles destacaram o fato de que uma profundidade excessiva pode levar à diminuição da possibilidade de flotar inclusões.

\subsection{Comportamento do Fluxo}

A estrutura de fluxo, para o caso em que se emprega uma válvula tubular, na seção de simetria da alma é apresentada na Figura 7.a, 7.b e 7.c. Para estas mesmas condições observa-se que o vórtice na região da alma ocorre na parte inferior do molde (distâncias superiores a $80 \mathrm{~cm}$ abaixo do menisco) em virtude da força do jato proveniente da válvula. $O$ jato retorna pelas laterais do flange oposto, subindo em direção ao menisco, em seguida ele se desloca no sentido da válvula e desce acompanhando o jato. No Plano 1 (Figuras 7.d, 7.e, 7.f), que passa pelo centro da válvula, observa-se a formação de quatro vórtices, dois na região inferior e outros dois próximo a válvula, e no Plano 2 (Figuras 7.h, 7.i, 7.j) também se verifica a formação de um vórtice na região superior.
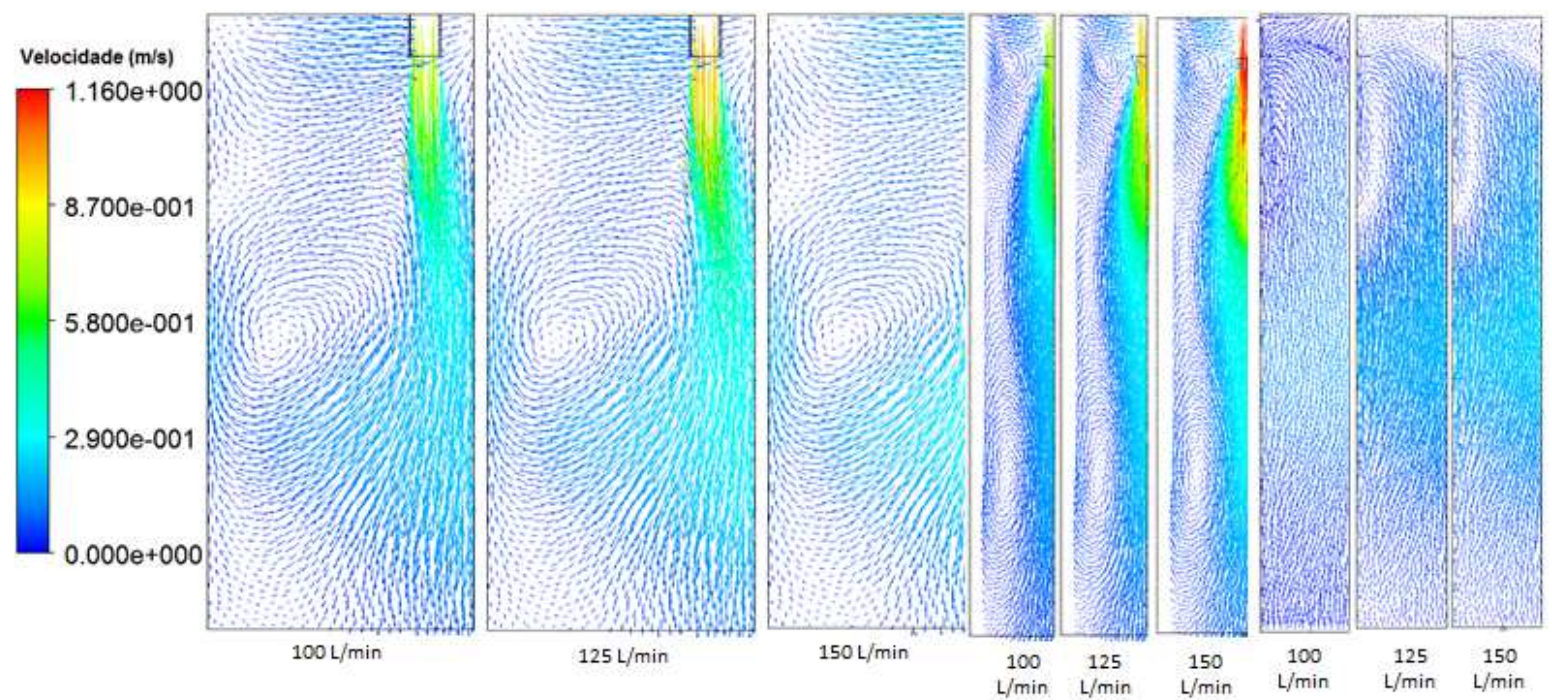

Figura 7. Mapa vetorial de velocidades obtido por simulação matemática para as vazões de 100,125 e $150 \mathrm{~L} / \mathrm{min}$ a), b) e c) vista da seção de simetria, d), e) e f) vista da metade do plano que corta a válvula, h), i) e j) Vista da metade do plano oposto .

A figura 8 mostra a evolução do fluxo obtida em filmagem na qual se acompanhou o movimento de particulas através da iluminação de um plano passando no centro da alma com laser, permitindo avaliar qualitativamente o fluxo do jato nesta região. A imagem é apresentada em escala de cores invertidas. Com base nas imagens, verifica-se que após doze segundos se tem uma completa evolução do jato, o que se caracteriza pelo total espalhamento das particulas ao longo da seção analisada, nesta imagem também é possível visualizar formação do vórtice apresentado na Figura 5. Pela figura 8, nota-se que o fluxo proveniente da válvula desce a distâncias superiores a $80 \mathrm{~cm}$ antes de retornar na região da alma e subir em direção ao menisco, passando pelo flange oposto. 


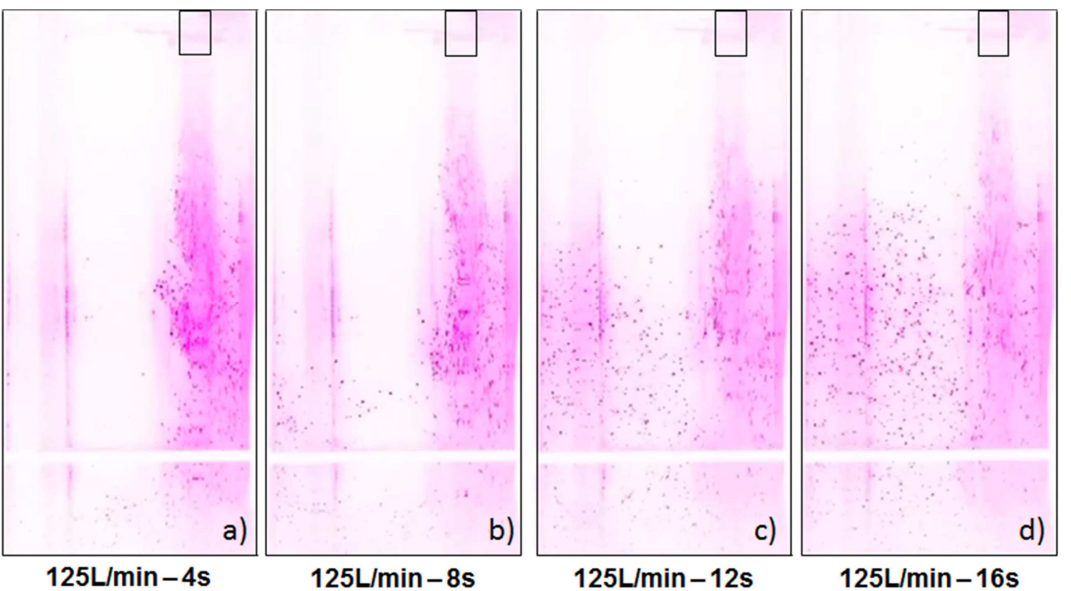

Figura 8. Visão do movimento das partículas dispersas no fluxo em molde de Beam Blank através da iluminação das mesmas por um plano de laser, para vazão de $125 \mathrm{~L} / \mathrm{min}: a) t=4 \mathrm{~s} ; \mathrm{b}) \mathrm{t}=8 \mathrm{~s}, \mathrm{c}) \mathrm{t}=12 \mathrm{~s}$ e d) $\mathrm{t}=16 \mathrm{~s}$,

No menisco houve a formação de quatro vórtices, sendo dois próximos da válvula e dois na quina do flange ao lado em que a mesma foi posicionada. No flange oposto o fluxo do fluido se movimenta das quinas para a região central, passa pela alma, desloca-se para a região do flange e em seguida desce acompanhando o jato.

Numa comparação quantitativa na região do menisco entre o fluxo com apenas uma válvula tubular e o fluxo com duas válvulas tubulares para velocidade de lingotamento de $0,98 \mathrm{~m} / \mathrm{min}$, percebe-se que não houve variação significativa nos valores de velocidade no menisco, sendo os valores máximos encontrados de $0,15 \mathrm{~m} / \mathrm{s}$ e $0,17 \mathrm{~m} / \mathrm{s}$, respectivamente. Para a primeira configuração a velocidade máxima ocorre na região que vai da alma até os filetes junto à válvula Figuras 9.a e 9.c, enquanto que na simulação com duas válvulas o valor máximo ocorre nos quatro filetes Figura 9.d, já no centro da alma encontrou-se valores de até $0,09 \mathrm{~m} / \mathrm{s}$ Figura 9.b. Chen et al [6] destacaram que velocidades relativamente baixas no menisco podem dificultar a fusão do pó fluxante; segundo De Santis et al [3] o limite de velocidade na superfície livre é $0,3 \mathrm{~m} / \mathrm{s}$.
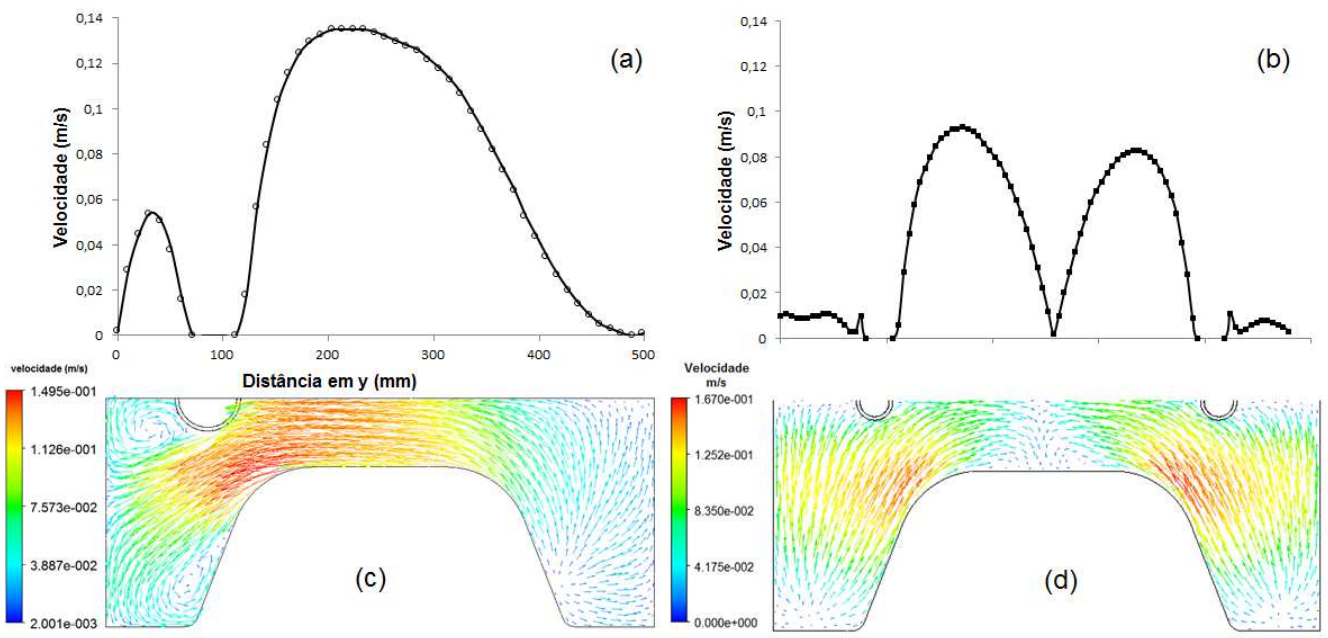

Figura 9. Gráfico de velocidade no menisco na linha de simetria para válvula: (a) Tipo A e (b) Tipo B. Mapa vetorial na região do menisco para válvula: (c) Tipo A e (d) Tipo B. Vazão de 125L/min

Valores altos de velocidade entre o filete e a válvula podem acarretar na diminuição da espessura da casca solidificada nesta região. Conforme previsto por Yang et al 
[2], a região do filete tende a apresentar menor espessura da casca solidificada. No caso da utilização de apenas uma válvula tubular, a assimetria do fluxo evidenciada na Figura 9.c sugere diferentes espessuras de pele junto aos filetes, em função de diferentes valores de velocidade.

\section{CONCLUSÃO}

Os valores de profundidade do jato para a configuração com apenas uma válvula tubular de diâmetro igual a 53,2 mm são maiores do que quando se utiliza duas válvulas tubulares com diâmetro interno de $26,7 \mathrm{~mm}$.

Com relação ao comportamento do fluxo observa-se a formação de quatro vórtices no menisco, um na alma, quatro no flange ao lado da válvula e um no flange oposto.

Utilizando somente uma válvula tubular, não houve variação significativa na velocidade quando comparado a utilização de duas válvulas tubulares.

A variação de velocidade na região do menisco pode causar uma menor espessura da casca dos filetes situados ao lado da válvula.

Os resultados obtidos através de simulação computacional foram coerentes com os resultados de simulação física.

No flange oposto as velocidades são menores do que as obtidas no lado em que se tem a válvula.

Os maiores valores de velocidades no flange oposto estão situados na parte inferior do mesmo.

\section{Agradecimentos}

Os autores gostariam de agradecer à Coordenação de Aperfeiçoamento de Pessoal de Nível Superior (CAPES), ao Conselho Nacional do Desenvolvimento Científico e Tecnológico (CNPq), e à Fundação Gorceix pelo apoio financeiro, em especial pelas bolsas de pesquisa concedidas a W.V. Gabriel, J. J. M. Peixoto, L. Q. Ribeiro e S. S. de Souza, respectivamente. Agradecem também à FAPEMIG pelo apoio.

\section{REFERÊNCIAS}

1 Chen W, Zhang YZ, Zhang CJ, Zhu LG, Zhang CJ, Chen Y, Wang BX, Wang C. Three-dimensional FEM study of fluid flow in mould for beam blank Continuous Continuous Casting: influence of nozzle structure and parameters on fluid flow. Ironmaking and Steelmaking. 2012;39(8):560-567.

2 Yang J-w, Du Y-p; Shi R, Cui X-c, Liu C. Effect of Sem Parameters on 3D Flow Field in mould of Beam Blank Continuous Casters. Iron and Steel . 2004; 11(6),

3 De Santis M; Cristallini A; Rinaldi M; Sgro, A. Modelling-based Innovative Feeding Strategy for Beam Blanks Mould Casting Aimed at As-cast Surface Quality Improvement. ISIJ International. 2014; 54(3), 496 - 503.

4 Lee J-E, Yoon J-K, Han HN. 3-dimensional mathematical model for the analysis beamblank casting using body fitted coordinate of continuous system. ISIJ International. 1998; 38(2):132-141

5 Hibbeler LC, Xu K, Thomas B.G, Koric S, Spangler C. Thermomechanical Modeling of beam blank Casting. Iron \& steel technology. 2009; 6(7):60-73.

6 Chen W, Zhang YZ, Zhu L-G, Zhang C-J, Chen Y, Wang BX, Wang C. Three dimensional FEM study of fluid flow in mould for beam blank continuous casting: influence of straight through conduit type SEN. Ironmaking and Steelmaking. 2012; 39(8):551-559. 